\title{
Berberine and Evodiamine Act Synergistically Against Human Breast Cancer MCF-7 Cells by Inducing Cell Cycle Arrest and Apoptosis
}

\author{
JIA DU ${ }^{1}$, YANG SUN ${ }^{1}$, YI-YU LU1, ERIC LAU ${ }^{2}$, MING ZHAO ${ }^{1,3}$, QIAN-MEI ZHOU ${ }^{1}$ and SHI-BING SU ${ }^{1}$ \\ ${ }^{1}$ Research Center for Traditional Chinese Medicine Complexity System, Shanghai \\ University of Traditional Chinese Medicine, Shanghai, P.R. China; \\ ${ }^{2}$ Cancer Center, Sanford-Burnham Medical Research Institute, La Jolla, CA, U.S.A.; \\ ${ }^{3}$ AntiCancer Inc., San Diego, CA, U.S.A.
}

\begin{abstract}
Background/Aim: The synergistic combinations of natural products have long been the basis of Traditional Chinese herbal Medicine formulas. In this study, we investigated the synergistic effects of a combination of berberine and evodiamine against human breast cancer MCF-7 cells in vitro and in vivo, and explored its mechanism. Materials and Methods: Cell survival was measured using the MTT assay. Apoptosis-related proteins were observed using western blot analysis. Apoptosis was detected with flow cytometric analysis and by Hoechst 33258 staining. Tumor xenografts were used in vivo. Results: Compared to berberine or evodiamine treatments alone, the combination treatment of berberine $(25 \mu M)$ and evodiamine $(15 \mu M)$ synergistically inhibited the proliferation of $M C F$ 7 cells in a time-dependent manner and resulted in the $G_{0} / G_{1}$ phase accumulation of cells that exhibited increased expression levels of the CDK inhibitors p21 and p27 with a concomitant reduction in the expression levels of cell-cycle checkpoint proteins cyclin D1, cyclin E, CDK4, and CDK6. Furthermore, the combination treatment induced apoptosis that was accompanied by increased expression levels of p53 and Bax, reduced expression levels of $\mathrm{Bcl}-2$, activation of
\end{abstract}

Correspondence to: Shi-Bing Su, Research Center for Traditional Chinese Medicine Complexity System, Shanghai University of Traditional Chinese Medicine, Shanghai 201203, P.R. China. Tel/Fax: +86 02151323013, e-mail: shibingsu07@163.com; Qianmei Zhou, Research Center for Traditional Chinese Medicine Complexity System, Shanghai University of Traditional Chinese Medicine, Shanghai 201203, P.R. China. Tel/Fax: +86 02151323013,e-mail: tazhou@163.com; Ming Zhao, Anticancer Inc., San Diego, CA, 92111, U.S.A., Phone/Fax: +1 8586542555, e-mail: mingz@hotmail.com

Key Words: Breast cancer, MCF-7 cell, berberine, evodiamine, synergistic combination. caspase-7, and caspase-9, and the cleavage of PARP. The combination of berberine and evodiamine synergistically inhibited tumor growth in vivo in MCF-7 human breast cancer xenografts. Conclusion: Combination of berberine and evodiamine acts synergistically to suppress the proliferation of MCF-7 cells by inducing cell cycle arrest and apoptosis, illustrating the potential synergistic and combinatorial application of bioactive natural products.

Worldwide, breast cancer is the most common cancer and is the leading cause of cancer deaths among women (1). Although great efforts have been made for the prevention and treatment of breast cancer, much progress remains to be made. It is necessary to identify superior strategies for breast cancer treatment to achieve sustained responses and more favorable outcomes for patients. Natural products are valuable sources of novel anti-cancer drugs due to their diverse biological activities and relatively low toxicities (2). Multi-drug therapy is currently being practiced worldwide for the treatment of numerous types of cancers, that inherently exhibit complex pathologies (3). Thus, the therapeutic application of natural products can synergistically improve the treatment of breast cancer.

Synergistic multi-drug combinations have long been the basis of traditional Chinese herbal medicine formulas, that represent a form of Chinese herbology in which herbs are combined to produce enhanced efficacy compared to the application of individual herbs (4). Recently, berberine and evodiamine, predominant compounds contained in the traditional Chinese herbal medicine formula Zuo-Jin-Wan, were shown to inhibit the proliferation of cancer cells. However, whether berberine and evodiamine elicit synergistic anti-proliferative effects against human breast cancer cells has been unclear. Thus, in this study, we evaluated the potential synergistic effects of berberine and evodiamine against human breast cancer MCF-7 cells. 
Zuo-Jin-Wan (ZJW) consists of two traditional Chinese medicinal herbs, Rhizoma Coptidis and Fructus Evodiae, and was experimentally demonstrated to elicit anti-cancer activity in gastric cancer (5), liver cancer (6), colorectal cancer (7), and multiple cancer cell lines. The anti-cancer activities of ZJW have been suggested to be attributed to the induction of the mitochondria-dependent apoptosis pathway (8). In our previous study, we investigated the effects and safety of Zuo-Jin-Wan (ZJW) using the 4T1 mouse breast cancer model. Our results showed that ZJW inhibits tumor growth and improves kidney and liver functions in the 4T1 breast cancer mice (9).

The compounds berberine and evodiamine, that represent the predominant components of Rhizoma Coptidis and Fructus Evodiae, respectively, have been demonstrated to exhibit significant bioactive functions. Berberine has been widely used in Asian countries for the treatment of diabetes, hypertension, and hypercholesterolemia (10-12). Evodimiane has been shown to exhibit diverse pharmacological activities against obesity and Influenza A Virus, to protect against myocardial ischemia-reperfusion injury, and to regulate testosterone secretion (13-16). Recent studies have shown that both evodiamine and berberine individually exhibit antitumor activities against a broad range of human cancer cells types; they have been shown to inhibit cancer cell proliferation and to induce apoptosis in prostate cells (1718). Moreover, berberine or evodiamine individually have been shown to decrease levels of Bcl-2, Bcl-xL, and Mcl-1 and to trigger apoptosis $(6,19-22)$ and have also been reported as potent inhibitors of nuclear factor $\mathrm{kB}(\mathrm{NF}-\mathrm{kB})$ mediated transcription (22).

The aim of this study was to determine a scientific rationale for the therapeutic efficacy of Traditional Chinese herbal medicine formula-based combinations of natural products. We investigated the anti-tumor efficacy and mechanisms of action elicited by a combination treatment of berberine and evodiamine in human breast cancer MCF-7 cells through in vivo and in vitro studies. Our results showed that berberine and evodiamine act synergistically against human breast cancer MCF-7 cells by inducing cell-cycle arrest and apoptosis. Our study demonstrates the potential synergistic therapeutic potential of formula-based combinations of bioactive natural products and might significantly influence the development of new therapeutic strategies in the treatment of cancer.

\section{Materials and Methods}

Chemicals. The compound evodiamine was obtained from the Shanghai Research and Development Centre for Standardization of Traditional Chinese Medicine (Shanghai, P.R. China), and berberine hydrochloride was purchased from Shanghai Tauto Biotech Co., Ltd (Shanghai, P.R. China). Stock solutions of evodiamine and berberine were prepared in dimethylsulfoxide (DMSO), stored at $4^{\circ} \mathrm{C}$, and diluted with fresh medium immediately prior to application. An equal volume of DMSO (final concentration $\leq 0.1 \%$ ) was added to the controls.

Cell culture. MCF-7 cells were obtained from the Chinese Academy of Sciences (Shanghai, China) and were cultured in DMEM medium (Gibco, San Francisco, CA, USA) supplemented with $10 \%$ heatinactivated $\left(56^{\circ} \mathrm{C}, 30 \mathrm{~min}\right)$ fetal calf serum (ExCell Biology, Shanghai, P.R. China), $0.01 \mathrm{mg} / \mathrm{mL}$ insulin (Sigma, St Louis, MO, USA), $2 \mathrm{mmol} / \mathrm{L}$ glutamine (Gibco, San Francisco, CA, USA), penicillin $(100 \mathrm{U} / \mathrm{ml})$ and streptomycin $(100 \mu \mathrm{g} / \mathrm{ml})$. The cells were incubated at $37^{\circ} \mathrm{C}$ in a humidified atmosphere with $5 \% \mathrm{CO}_{2}$.

Cell proliferation assay. Inhibition of cell growth was assessed by MTT assay. MCF-7 cells $\left(1 \times 10^{4}\right.$ cells/well $)$ were seeded into $96-$ well culture plates. After an overnight incubation, the MCF-7 cells were treated with the indicated concentrations of berberine, evodiamine, or berberine plus evodiamine (the concentration of each compound was to controls) Following incubation with the compounds, cell growth was measured at the indicated time points by the addition of $20 \mu \mathrm{l} \mathrm{MTT}$ at $37^{\circ} \mathrm{C}$ for $4 \mathrm{~h}$. Next, DMSO $(150 \mu \mathrm{l})$ was added to dissolve the formazan crystals. Optical density (OD) was measured at $490 \mathrm{~nm}$ using an ELISA plate reader (BioTek, Winooski, VT, USA).

Isobologram analysis of the efficacy of berberine and evodiamine. Isobologram analysis was used to determine the effects of drug combinations on the MCF-7 cells. The interaction of berberine and evodiamine was quantified by determining the combination index (CI), according to the following classic isobologram: $\mathrm{CI}=(\mathrm{D})_{1} /(\mathrm{Dx})_{1}$ $+(\mathrm{D})_{2} /(\mathrm{Dx})_{2}$, where $\mathrm{Dx}$ is the individual dose of berberine or evodiamine required to produce an effect, and (D) 1 and (D) $)_{2}$ are the respective doses of berberine and evodiamine that are necessary to produce the same effect in combination. From this analysis, the combined effects of the two drugs can be summarized as follows: the CI of less than, equal to, or more than 1 indicate synergistic, additive, or antagonistic effects, respectively.

Cellular morphological observation. MCF-7 cells $\left(2 \times 10^{5} / \mathrm{ml}\right)$ were seeded onto sterile coverslips that were placed into 6-well plates. After attachment to the coverslips, the MCF-7 cells were treated with $25 \mu \mathrm{mol} / \mathrm{l}$ berberine, $15 \mu \mathrm{mol} / 1$ evodiamine, or $25 \mu \mathrm{mol} / 1$ berberine plus $15 \mu \mathrm{mol} / \mathrm{l}$ evodiamine. After a 48 -h incubation, the cells were washed carefully with phosphate buffered saline (PBS), fixed with 4\% paraformaldehyde for $15 \mathrm{~min}$ at room temperature, washed with pre-chilled PBS 3 times and stained with $5 \mu \mathrm{g} / \mathrm{ml}$ Hoechst 33258 at room temperature in the dark for $20 \mathrm{~min}$. The samples were visualized using a fluorescent microscope (Olympus, Tokyo, Japan). Cells exhibiting cytoplasmic and nuclear shrinkage, chromatin condensation, or fragmentation were defined as apoptotic cells.

Cell cycle analysis. MCF- 7 cells $\left(4 \times 10^{5}\right.$ cells $\left./ \mathrm{ml}\right)$ were cultured in 60-mm dishes to $70-80 \%$ confluence. Cells were then treated with $25 \mu \mathrm{mol} / \mathrm{l}$ berberine, $15 \mu \mathrm{mol} / 1$ evodiamine, or $25 \mu \mathrm{mol} / 1$ berberine plus $15 \mu \mathrm{mol} / \mathrm{l}$ evodiamine for $48 \mathrm{~h}$. The cells were harvested and washed twice with PBS, then fixed with $70 \%$ ethanol at $-20^{\circ} \mathrm{C}$ for $24 \mathrm{~h}$. The cells were then collected and resuspended in PBS with RNase $(25 \mu \mathrm{g} / \mathrm{ml})$ and propidium iodide (PI, $50 \mu \mathrm{g} / \mathrm{ml})$ for $30 \mathrm{~min}$ at $37^{\circ} \mathrm{C}$. The PI-stained cells were analyzed using Mcycle software (Beckman Coulter, Fullerton, CA, USA). 

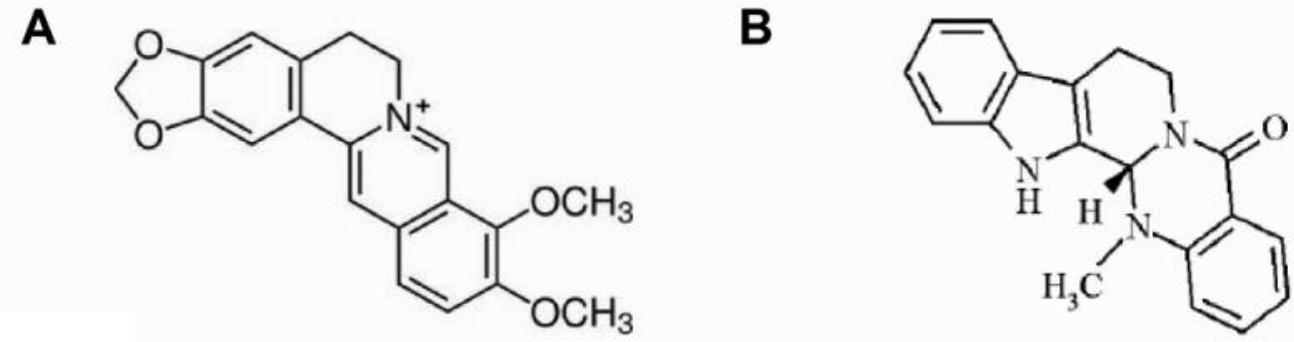

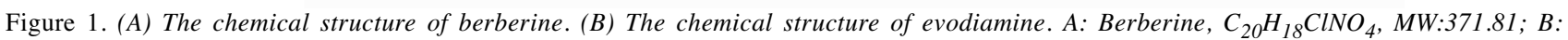
Evodiamine, $\mathrm{C}_{19} \mathrm{H}_{17} \mathrm{~N}_{3} \mathrm{O}$, MW:303.36.

Annexin V-PI staining assay. MCF-7 cells $\left(1 \times 10^{6}\right.$ cells $\left./ \mathrm{mL}\right)$ were cultured in $100-\mathrm{mm}$ dishes to $70-80 \%$ confluence. The cells were then treated with $25 \mu \mathrm{mol} / \mathrm{l}$ berberine, $15 \mu \mathrm{mol} / \mathrm{l}$ evodiamine, or 25 $\mu \mathrm{mol} / \mathrm{l}$ berberine plus $15 \mu \mathrm{mol} / \mathrm{l}$ evodiamine for $48 \mathrm{~h}$. The cells were collected and evaluated using an Annexin V-FITC/PI Apoptosis Kit (Beckman Coulter, Miami, FL, USA), according to the manufacturer's protocols. The cells were analyzed using a flow cytometer (Beckman Coulter, Fullerton, CA, USA). The percentage of apoptotic cells was determined using Mcycle software.

Western blot analysis. MCF-7 cells $\left(1 \times 10^{6}\right.$ cells/dish in a $100-\mathrm{mm}$ dish) were treated with $25 \mu \mathrm{mol} / \mathrm{l}$ berberine, $15 \mu \mathrm{mol} / \mathrm{l}$ evodiamine, or $25 \mu \mathrm{mol} / \mathrm{l}$ berberine plus $15 \mu \mathrm{mol} / \mathrm{L}$ evodiamine for $48 \mathrm{~h}$. Total cell lysates were generated using RIPA lysate buffer containing $1 \mathrm{mM}$ PMSF and boiled with $5 \mathrm{X}$ sample loading buffer. The protein concentration of each lysate was determined by using the BCA method. Lysates $(30-50 \mu \mathrm{g})$ were subjected to $8-12 \%$ SDS-PAGE. The proteins were transferred onto PVDF membranes, and the membranes were incubated with blocking buffer (5\% non-fat dry milk in TBS- $0.1 \%$ Tween 20 ) for $1 \mathrm{~h}$ at room temperature. The membranes were then incubated with the indicated antibodies overnight at $4^{\circ} \mathrm{C}$. The membranes were incubated with corresponding IRDye $800 \mathrm{CW}$ or $680 \mathrm{CW}$-conjugated secondary antibodies for $2 \mathrm{~h}$ at room temperature. The proteins were detected using an Odyssey infrared imaging system (Li-Cor Bioscience, Lincoln, NE), and the protein bands were subjected to densitometric analysis and shown as the ratio of the intensity of the target protein relative to that of GAPDH.

Tumor xenograft and treatment. Athymic female BALB/c mice (5 weeks of age) were obtained from the Academia Sinica (Shanghai, China). Five mice were randomly distributed into the normal group. Other mice were assigned to the tumor xenograft groups and treated as follows: prior to inoculation, $17 \beta$-estradiol was intraperitoneally injected, and then the MCF-7 cells $\left(1 \times 10^{7}\right.$ cells $\left./ \mathrm{ml}\right)$ were inoculated into the mammary fat pad (m.f.p.) of the mice. Once palpable tumors developed (approximately 2 weeks), the tumor-bearing mice were randomized to 4 groups ( $n=5$ per group). Treatment groups included berberine $(10 \mathrm{mg} / \mathrm{kg})$, evodiamine $(10 \mathrm{mg} / \mathrm{kg})$, and a combination of berberine $(10 \mathrm{mg} / \mathrm{kg})$ and evodiamine $(10 \mathrm{mg} / \mathrm{kg})$. All of the compounds were administrated intraperitoneally once every 2 days. Untreated mice were divided into normal (no MCF-7 xenograft) and control groups (xenografted MCF-7 cells) that were injected with physiological saline. Tumor sizes were determined by measuring the largest length/width and perpendicular diameters every week. Tumor volumes were calculated according to the following formula: $\mathrm{V}=\mathrm{A} \times \mathrm{B}^{2} \times 0.52$, where $\mathrm{A}$ is the largest superficial diameter and $B$ is the smallest superficial diameter. During the treatment period, the mice of each group were also monitored for body weight, food intake, posture, and movement. After 5 weeks of treatment, the animals were sacrificed. All procedures conformed to Institutional regulations of animal welfare and were approved by the ethical committee of Shanghai University of traditional Chinese medicine.

Statistical analysis. All data are expressed as mean values \pm SD. Comparisons between groups were performed by Student's $t$-test and one-way analysis of variance (ANOVA). The level of significance was set at $p<0.05$.

\section{Results}

Combination of berberine and evodiamine synergistically inhibit the proliferation of MCF-7 cells. To investigate the antiproliferative effects of the combination of berberine and evodiamine, MCF-7 cells were treated with or without the indicated concentrations of berberine, evodiamine, or their combination for $48 \mathrm{~h}$. The MTT results showed that both berberine (Figure 1A) and evodiamine (Figure 1B) significantly inhibited cell viability in a concentrationdependent manner. The $50 \%$ inhibitory concentrations $\left(\mathrm{IC}_{50}\right)$ for berberine and evodiamine were 50 and $60 \mu \mathrm{M}$, respectively (Figures 2A and B). Next, the effect of berberine in combination with evodiamine was investigated. The dose of berberine was fixed at $25 \mu \mathrm{M}\left(1 / 2 \mathrm{IC}_{50}\right)$ and the dose of evodiamine was fixed at $15 \mu \mathrm{M}\left(1 / 4 \mathrm{IC}_{50}\right)$. We tested both the combination of berberine $(25 \mu \mathrm{M})$ with a range of concentrations of evodiamine and the combination of evodiamine $(15 \mu \mathrm{M})$ with a range of concentrations of berberine on MCF-7 cells (Figures $2 \mathrm{~A}$ and $\mathrm{B}$ ). After treatment with the combination of $25 \mu \mathrm{M}$ berberine plus $3.75 \mu \mathrm{M}$ evodiamine, cell growth was further inhibited by approximately $10 \%$ compared to $3.75 \mu \mathrm{M}$ evodiamine alone $(p<0.05)$. Similarly, after treatment with the combination of $25 \mu \mathrm{M}$ berberine plus $15,30,60$ and $120 \mu \mathrm{M}$ evodiamine respectively, 
cell growth was further inhibited by approximately $16,17,13$, $26 \%$ compared to $15,30,60,120 \mu \mathrm{M}$ evodiamine alone $(p<0.01)$. Cell growth inhibition did not significantly change when we combined $25 \mu \mathrm{M}$ berberine with $7.5 \mu \mathrm{M}$ evodiamine $(p>0.05$ ), compared to $7.5 \mu \mathrm{M}$ evodiamine alone (Figure $2 \mathrm{~A}$ ). Moreover, there were no significant differences between the effects elicited by $25 \mu \mathrm{M}$ berberine plus $15 \mu \mathrm{M}$ evodiamine and $25 \mu \mathrm{M}$ berberine plus $30 \mu \mathrm{M}$ evodiamine $(p>0.05)$.

After a combination treatment of $15 \mu \mathrm{M}$ evodiamine plus $3.125 \mu \mathrm{M}$ berberine, $15 \mu \mathrm{M}$ evodiamine plus $6.25 \mu \mathrm{M}$ berberine, $15 \mu \mathrm{M}$ evodiamine plus $25 \mu \mathrm{M}$ berberine and $15 \mu \mathrm{M}$ evodiamine plus $100 \mu \mathrm{M}$ berberine, cell growth was inhibited by approximately $23,22,13$, and $5 \%$, compared to $3.125,6.25,25$ or $100 \mu \mathrm{M}$ berberine alone, respectively $(p<0.05$ or $p<0.01)$. However, $15 \mu \mathrm{M}$ evodiamine plus 12.5 or $50 \mu \mathrm{M}$ berberine did not change the effect on cell survival $(p>0.05)$, as compared to 12.5 or $50 \mu \mathrm{M}$ berberine alone (Figure 2B). There were no significant differences between $15 \mu \mathrm{M}$ evodiamine plus $25 \mu \mathrm{M}$ berberine and $15 \mu \mathrm{M}$ evodiamine plus $50 \mu \mathrm{M}$ berberine $(p>0.05)$.

Isobologram analysis revealed a $\mathrm{CI}<1$ for $25 \mu \mathrm{M}$ berberine in combination with 15,60 , or $120 \mu \mathrm{M}$ evodiamine, respectively, indicating a synergistic relationship between the treatments (Table I). Moreover, the combination treatment of $15 \mu \mathrm{M}$ evodiamine and 25 or $50 \mu \mathrm{M}$ berberine also indicated synergistic relationship between the treatments $(\mathrm{CI}<1)$ (Table II). The combination of $25 \mu \mathrm{M}$ berberine and $15 \mu \mathrm{M}$ evodiamine created a synergistic effect $(\mathrm{CI}=0.75)$. Furthermore, the concentration of this combination was lowest compared to the concentrations of all of the other synergistic combinations. Therefore, $25 \mu \mathrm{M}$ berberine combined with $15 \mu \mathrm{M}$ evodiamine was selected for the subsequent experiments.

Combination of berberine and evodiamine enhances apoptosis of MCF-7 cells. We investigated the rate of apoptosis of MCF-7 cells by morphological evaluation after combination treatment with berberine and evodiamine for $48 \mathrm{~h}$. The images showed that MCF-7 cells underwent apoptosis when treated with the combination of berberine with evodiamine. Hoechst 33258 staining showed that MCF-7 cells in the control group exhibited uniform chromatin and regular nuclear morphology. After treatment with the combination of berberine and evodiamine, typical features of apoptosis including cytoplasmic and nuclear shrinkage, chromatin condensation, formation of apoptotic bodies and smaller cellular bodies, cell volume shrinkage, intracellular vacuole formation, chromatic agglutination, and chromatorrhexis were observed at the indicated time points (Figure 3A). To determine the apoptotic ratio induced by the compound combinations, apoptosis was analyzed by flow cytometry after MCF-7 cells were exposed to $25 \mu \mathrm{M}$ berberine, $15 \mu \mathrm{M}$ evodiamine or $25 \mu \mathrm{M}$ berberine plus $15 \mu \mathrm{M}$ evodiamine for $48 \mathrm{~h}$. The combination treatments
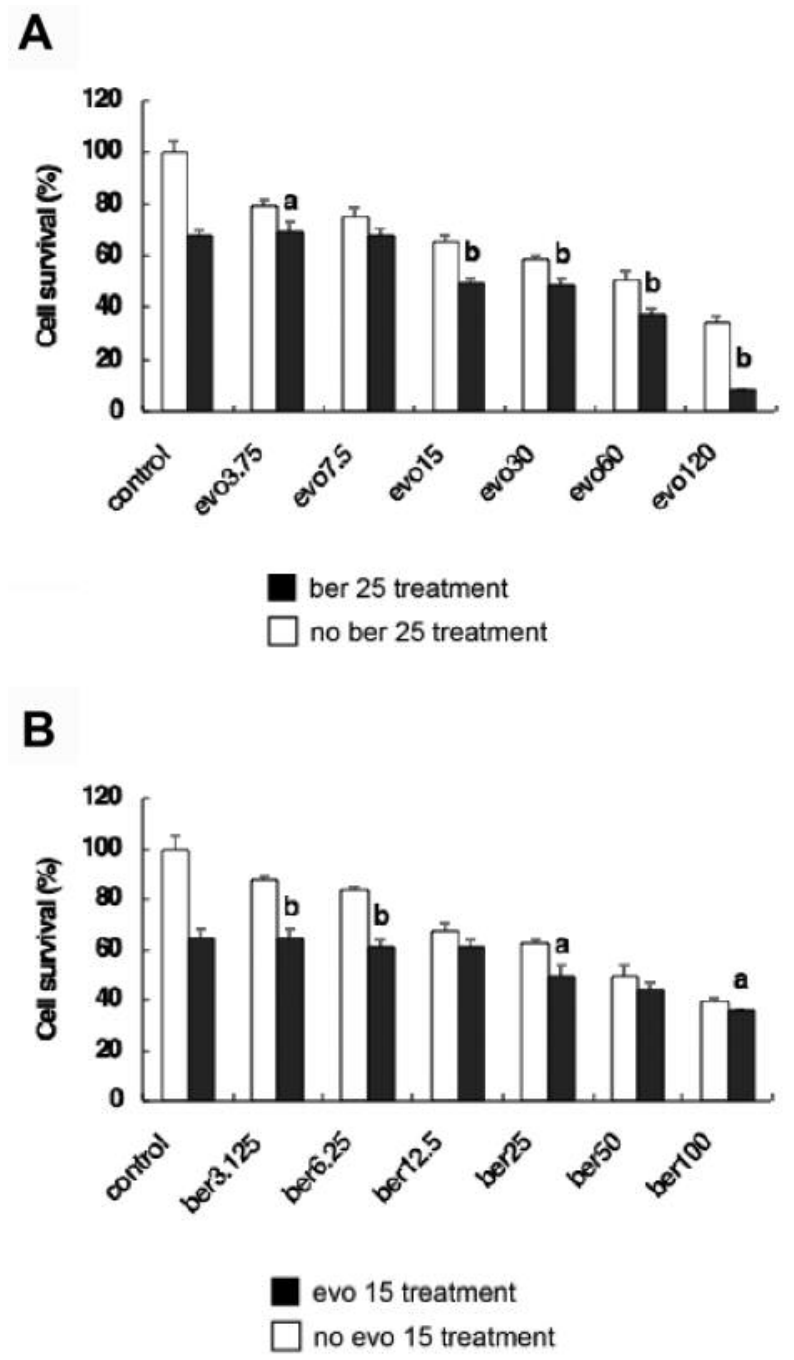

Figure 2. The effect of berberine (ber), evodiamine (evo), or their combination on the viability of MCF-7 cells. (A) MCF-7 cells were treated with $3.75,7.5,15,30,60,120 \mu \mathrm{M}$ evodiamine with (black columns) or without (white columns) $25 \mu \mathrm{M}$ berberine for $48 \mathrm{~h}$. $a<0.05, b<0.01$ (B) MCF-7 cells were treated with 3.125, 6.25, 12.5, $25,50,100 \mu M$ berberine with (black columns) or without (white columns) $15 \mu M$ evodiamine for $48 h . a<0.05, b<0.01$. Values are expressed mean values $\pm S D$. Data shown represent experiments performed in biological triplicates.

significantly increased apoptosis $(p<0.01$ ) (by 20 and $32 \%$, respectively) compared to either drug alone; when berberine and evodiamine were applied individually, only 29.1 and $17.1 \%$ of the cells exhibited apoptotic features at $48 \mathrm{~h}$, respectively (Figures 3B and C).

Combined berberine and evodiamine induces cell-cycle arrest of MCF-7 cells. The combination of berberine and evodiamine not only enhanced apoptosis, but also induced 

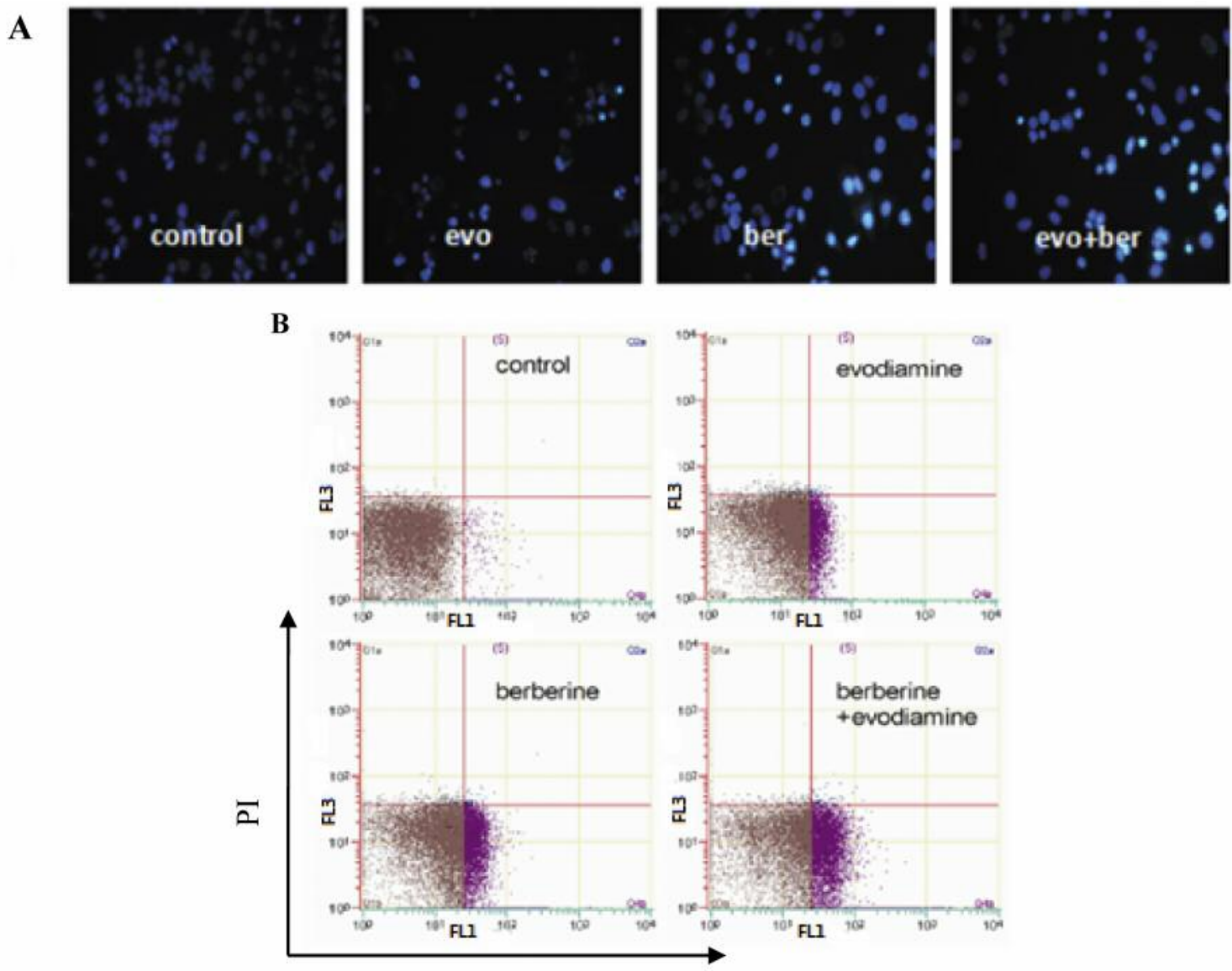

Annexin V-FITC

C

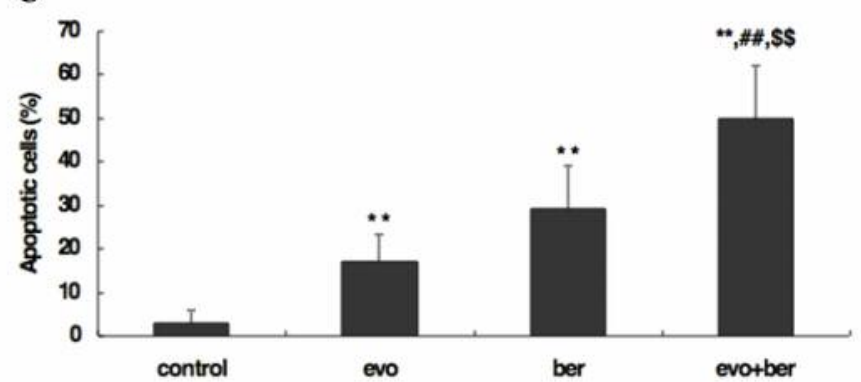

Figure 3. The effect of berberine (ber), evodiamine (evo), or their combination on apoptosis. (A) MCF-7 cells were treated with $25 \mu M$ ber, $15 \mu M$ evo or their combination for $48 \mathrm{~h}$. The cells were stained with Hoescht 33258 and visualized using a fluorescence microscope ( $\times 400)$. (B) MCF-7 cells were treated with $25 \mu M$ berberine, $15 \mu M$ evodiamine, or their combination for 48 h and analyzed by FACS. Representative flow cytometric profiles are shown. (C) The quantity of apoptotic cells was calculated. The data shown represent experiments performed in biological triplicates.

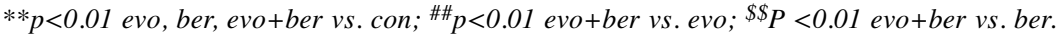

cell-cycle arrest. As shown in Figure 4, the combination treatment increased the fraction of cells in the $\mathrm{G}_{0} / \mathrm{G}_{1}$ phase by $15 \%$, as compared to the control treatment at $48 \mathrm{~h}$, suggesting that the combination of berberine and evodiamine induced a $G_{0} / G_{1}$ arrest. There were no significant differences between $15 \mu \mathrm{M}$ evodiamine plus $25 \mu \mathrm{M}$ berberine, and 15 $\mu \mathrm{M}$ evodiamine or $25 \mu \mathrm{M}$ berberine alone $(p>0.05)$. However, sub- $\mathrm{G}_{1}$ cells, which represent the apoptotic cells, increased significantly by 27 and $33 \%$ with the combination treatments, compared to either drug alone. 

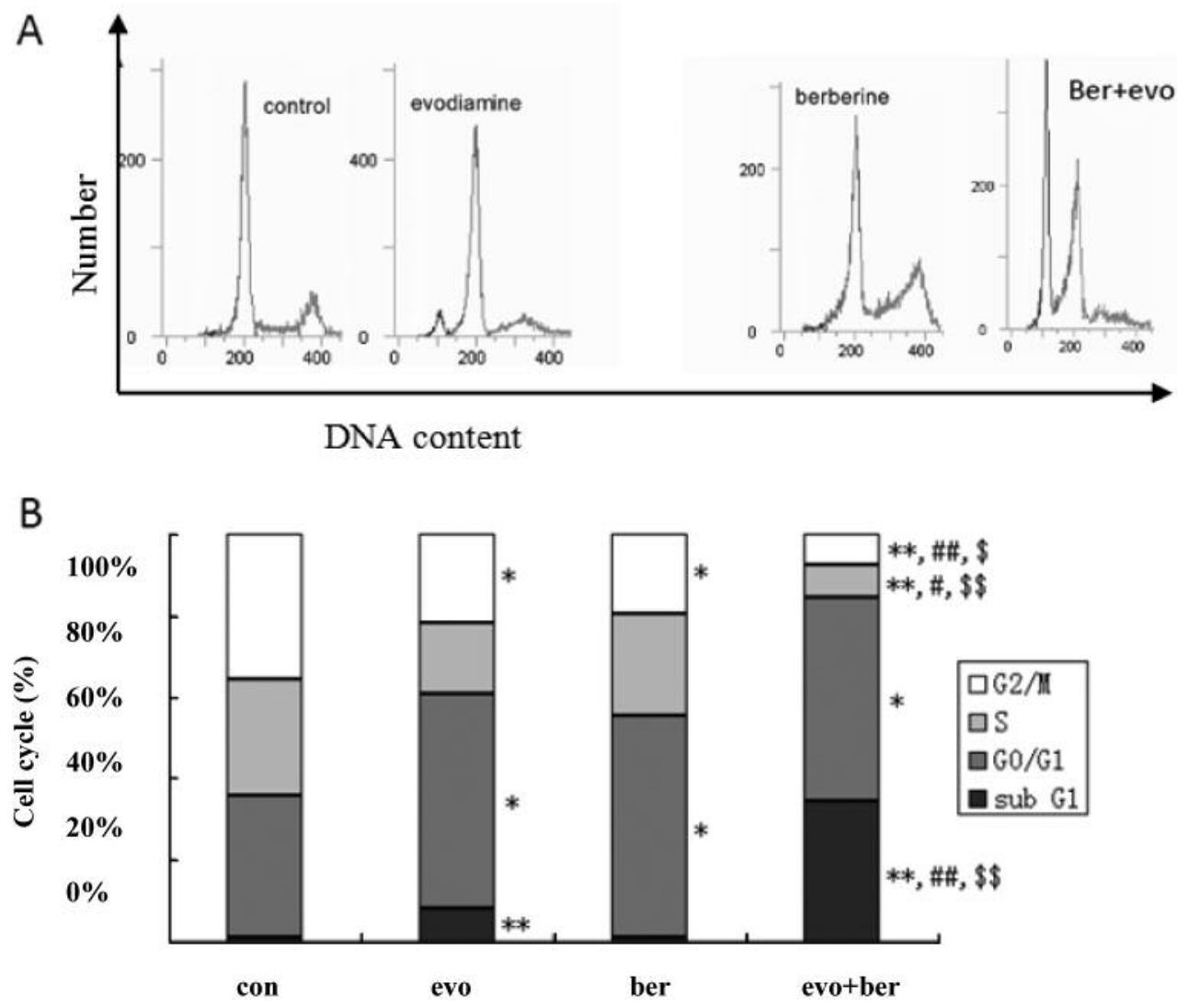

Figure 4. The effects of berberine (ber), evodiamine (evo), or combination on the cell-cycle progression of MCF-7 cells. MCF-7 cells were treated with $25 \mu \mathrm{M}$ berberine, $15 \mu \mathrm{M}$ evodiamine, or their combination for $48 \mathrm{~h}$. The MCF-7 cells were stained with PI and analyzed by flow cytometry. (A) Representative flow cytometric profiles. (B) The quantity of cells in different cell-cycle phase. Data shown represent experiments performed in biological triplicates. ${ }^{*} p<0.05,{ }^{* *} p<0.01$ evo, ber, evo + ber vs. con; ${ }^{\#} p<0.05,{ }^{\# \#} p<0.01$ evo + ber vs. evo, ${ }^{\$} p<0.05,{ }^{\$} p<0.01$ evo + ber vs. ber.

Table I. The CI of Evo 15 combination with different concentrations of Ber $(\mu M)$.

\begin{tabular}{lcccccc}
\hline Group & Evo+Ber 3.125 & Evo+Ber 6.25 & Evo+Ber 12.5 & Evo+Ber 25 & Evo+Ber 50 & Evo+Ber 100 \\
\hline CI & 1.208 & 1.178 & 1.418 & 0.75 & 0.834 & 1.079 \\
\hline
\end{tabular}

Table II. The CI of Ber 25 combination with different concentrations of Evo $(\mu M)$.

\begin{tabular}{lcccccc}
\hline Group & Ber+Evo 3.75 & Ber+Evo 7.5 & Ber+Evo 15 & Ber+Evo 30 & Ber+Evo 60 & Ber+Evo 120 \\
\hline CI & 2.586 & 1.536 & 0.75 & 0.966 & 0.75 & 0.525 \\
\hline
\end{tabular}

Effects of berberine and evodiamine on the levels of p21, p27, cyclin D1, cyclin E, and CDK 2, 4, 6 in MCF-7 cells. In mammalian cells, replication is regulated in an orderly fashion from $G_{1}$ to $S$ to mitosis by phase-specific oscillations in the level of Cyclins, Cyclin-dependent protein kinases
(CDKs), and CDK inhibitors. Cell-cycle progression is accelerated by cyclins and CDKs, and decelerated by CDK inhibitors (such as p16, p21 and p27), p53, retinoblastoma tumor suppressor protein (RB), and ARF. The D-type cyclins (cyclin D1, D2 and D3) are involved in regulation of the 

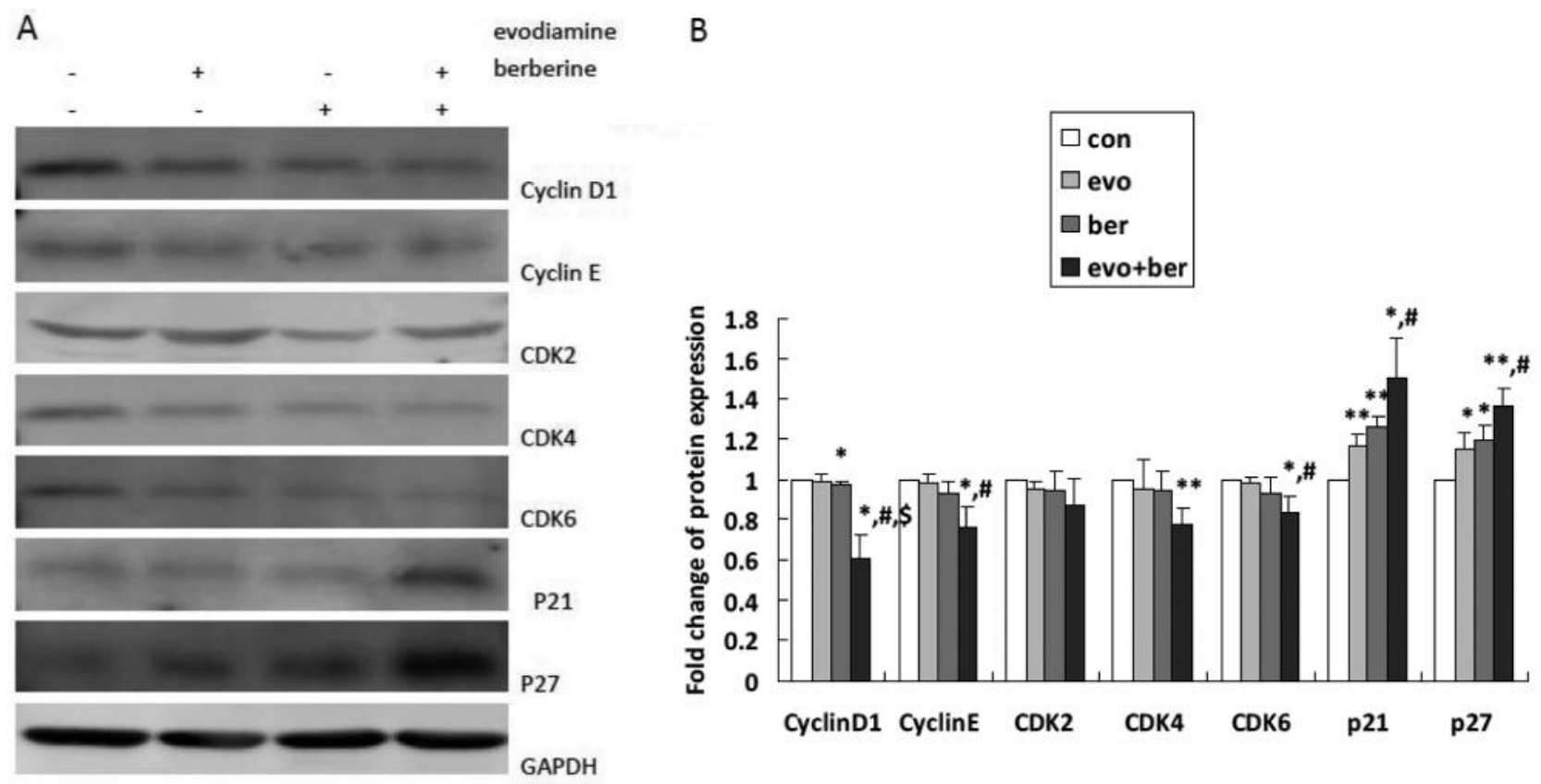

Figure 5. The expression levels of p21, p27, Cyclin D1, Cyclin E, CDK2, CDK4, and CDK6 as affected by $25 \mu M$ berberine (ber), $15 \mu M$ evodiamine (evo), or their combination on MCF-7 cells. (A) MCF-7 cells were treated with ber, evo, or ber plus evo for 48 h. The densitometric ratios of the indicated protein bands to GAPDH are shown as relative expression. The values are expressed as mean values $\pm S D, n=3 .{ }^{*} p<0.05$, **p<0.01, evo, ber, evo+ber vs. con. ${ }^{\#} p<0.05$, evo+ber vs. evo; ${ }^{\$} p<0.05$, evo+ber vs. ber.

G1/S transition. Their crucial function is to activate CDK4 and CDK6. (9-11) To elucidate the mechanisms underlying the cell-cycle arrest induced by the combination berberine and evodiamine in MCF-7 cells, we performed immunoblot analysis to evaluate p21/Waf1, p27- cell cycle inhibitors, cyclin D1, cyclin E, cyclin-dependent protein kinase 2 (CDK2), CDK4 and CDK6. The p21 and p27 levels increased after combination treatment. In contrast, the levels of cyclin D1, cyclin E, CDK4 and CDK6 decreased, whereas the combination treatment did not affect CDK2 expression $(p>0.05)$ (Figure 5).

Effects of berberine and evodiamine on the levels of p53, Bcl-2, Bax, caspase-7, -8, -9 and PARP in MCF-7 cells. To determine which proteins are regulated by the compound combination, we analyzed the protein levels of caspase-9, caspase-8, caspase-7, PARP, the Bcl-2 family including Bax and Bcl-2. As shown in Figure 6, caspase-9, caspase-7 and PARP cleavage products were observed upon treatment with the drug combination at $48 \mathrm{~h}$ in MCF-7 cells. The level of Bcl-2 decreased by the combined drug treatment at $48 \mathrm{~h}$. In contrast, the levels of Bax and p53 in MCF-7 cells increased by the combined drug treatment at $48 \mathrm{~h}$, as compared to the control treatment. However, the combination treatment did not affect caspase- 8 expression $(p>0.05)$. Apoptosis occurs either through the extrinsic pathway or the intrinsic pathway.
Efficacy of berberine and evodiamine on human breast cancer MCF-7 xenografts. To evaluate the anti-tumor effects of combined berberine and evodiamine in vivo, the experimental MCF-7 human breast cancer xenografts in nude mice were established to evaluate treatments with berberine (10 $\mathrm{mg} / \mathrm{kg})$, evodiamine $(10 \mathrm{mg} / \mathrm{kg})$, and their combined application. Mice with visible subcutaneous tumors were divided randomly into 4 groups. The tumor volume and body weight were measured every week. The average body weights of the control, evodiamine-, berberine- and combinationtreated mice did not differ significantly throughout the study period (Figure 7A). Movement, indigestion, and swelling appeared normal among the 3 groups of treated mice. When the experiment was terminated after 6 weeks, the tumor volumes and weights were significantly reduced by the combination treatment, compared with berberine or evodiamine treatments alone (Figures 7B and D).

\section{Discussion}

The treatment of cancer requires treatments with similar levels of complexity as its complex etiology (23). TCM formula-based combinations of natural products provide this type of complexity as their constituents elicit therapeutic effects via multi-targeted mechanisms to achieve synergistic efficacy (24). In this study, the combination treatment 


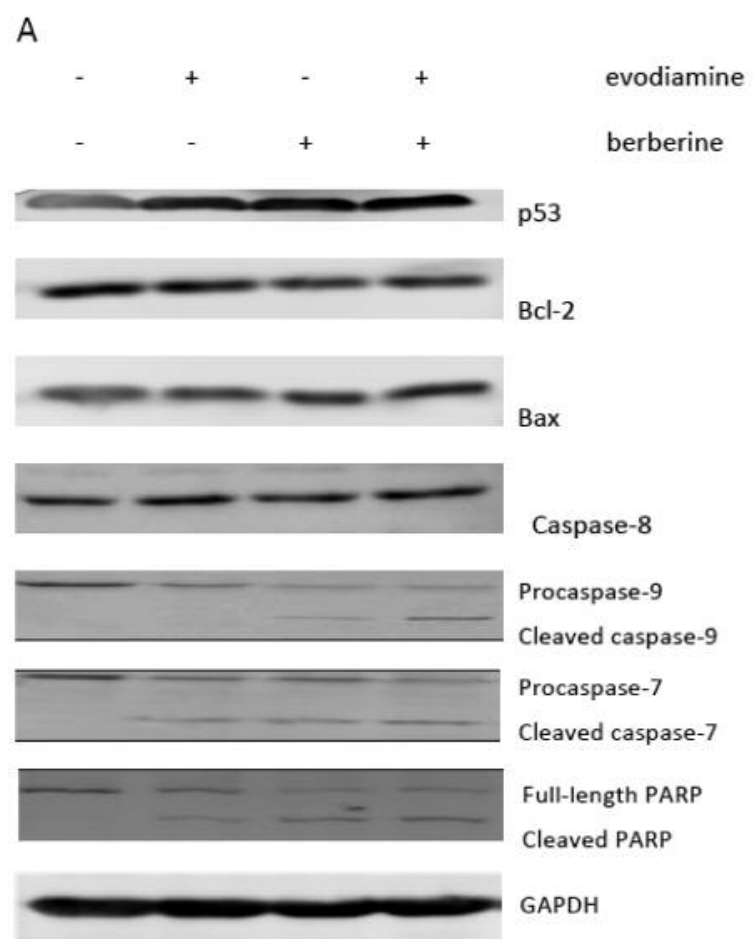

B

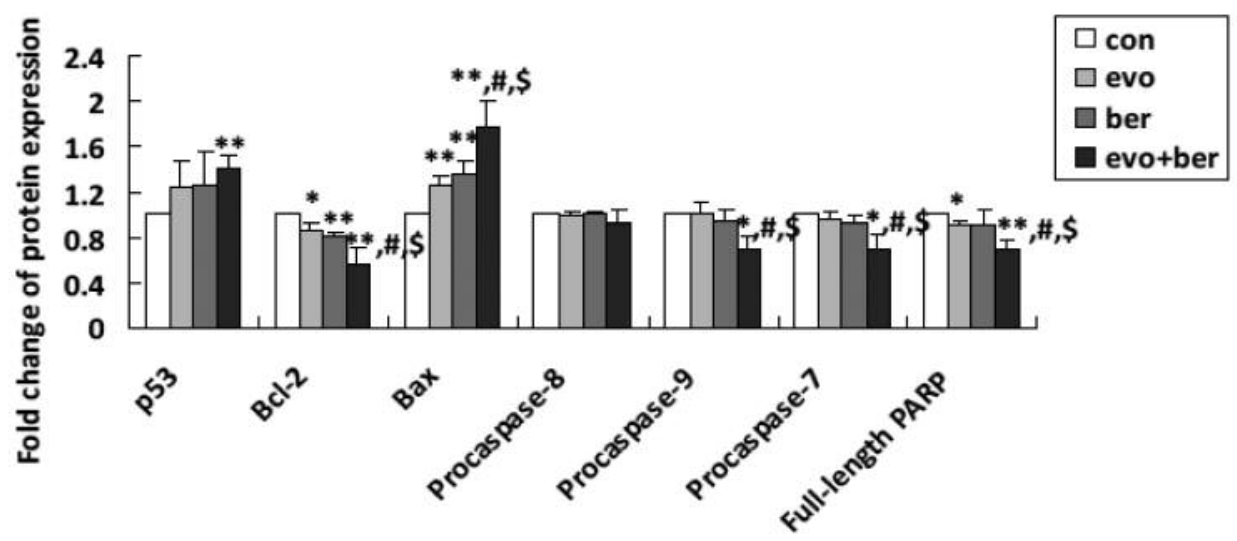

Figure 6. The expression levels of p53, Bcl-2, Bax, caspase-8, caspase-9, Caspase-7, and PARP as affected by 25 uM berberine (ber), evodiamine (evo) $15 \mu M$, and their combinatorial application. MCF-7 cells were treated with ber, evo, or ber plus evo for 48 h. The densitometric ratio of proteins relative to GAPDH is shown as relative expression values. Values are expressed as mean values $\pm S D, n=3 . * p<0.05$, ** $p<0.01$ evo, ber, evo+ber vs. administration; ${ }^{\#} p<0.05$, evo+ber vs. evo; ${ }^{\$} p<0.05$, evo+ber vs. ber.

investigated was synergistic and significantly inhibited cell growth of human breast cancer MCF-7 cells by inducing cell-cycle arrest and inducing apoptosis. This synergistic method showed particular effectiveness in targeting different cell death-inducing processes.

As shown in Figure 8, the combination of berberine and evodiamine induced cell-cycle arrest at $\mathrm{G}_{1}$ with multipletarget actions through up-regulation of p21, p27, and p53 and down-regulation of cyclin D1, cyclin E, CDK4, and CDK6. Our results suggest that caspase-9, caspase-7, and PARP are activated by combination treatment of berberine and evodiamine. This study showed that the down-regulation of Bcl-2 and up-regulation of Bax were triggered by the combination of berberine and evodiamine but not to treatment with berberine or evodiamine alone. The levels of p53 were up-regulated by the combined drug treatment, 
A

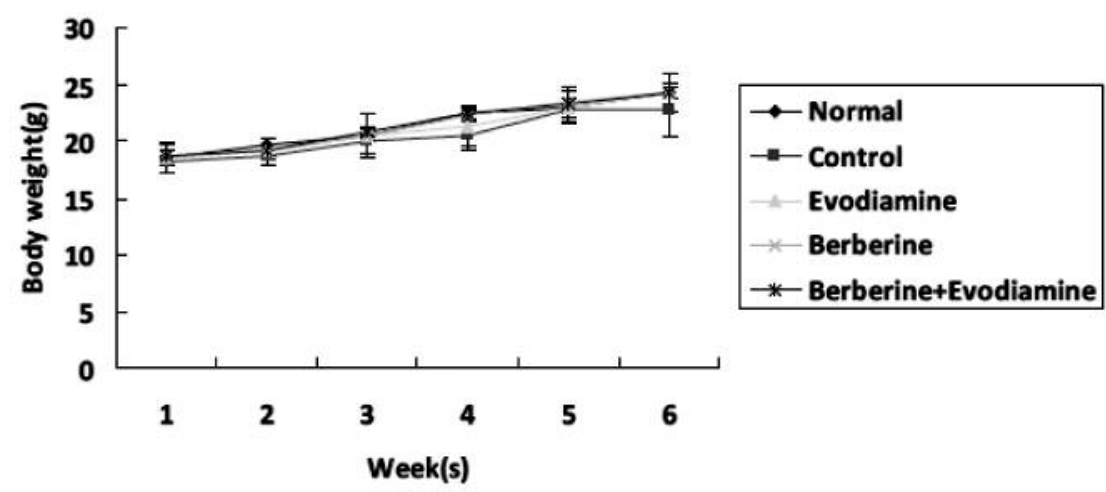

B

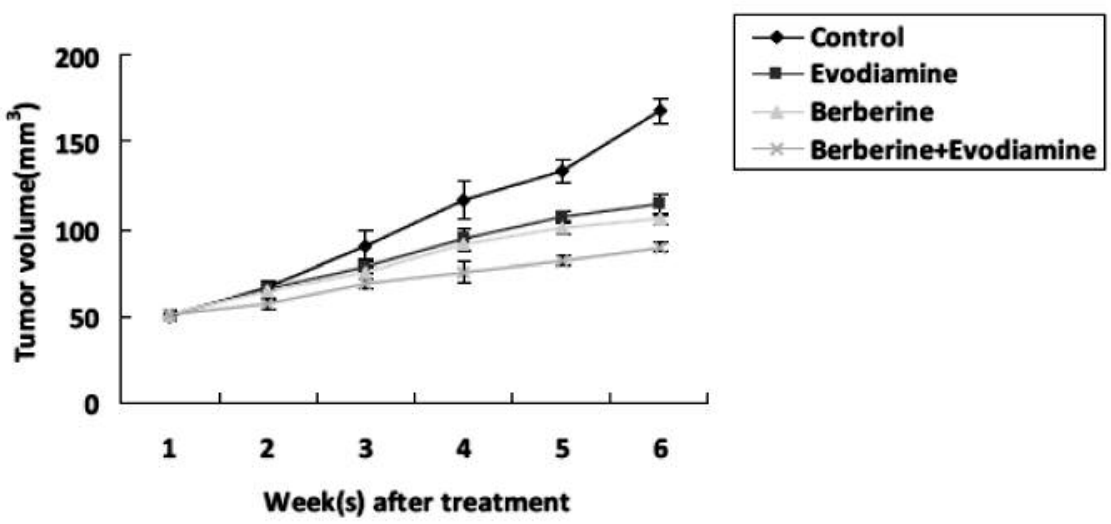

C

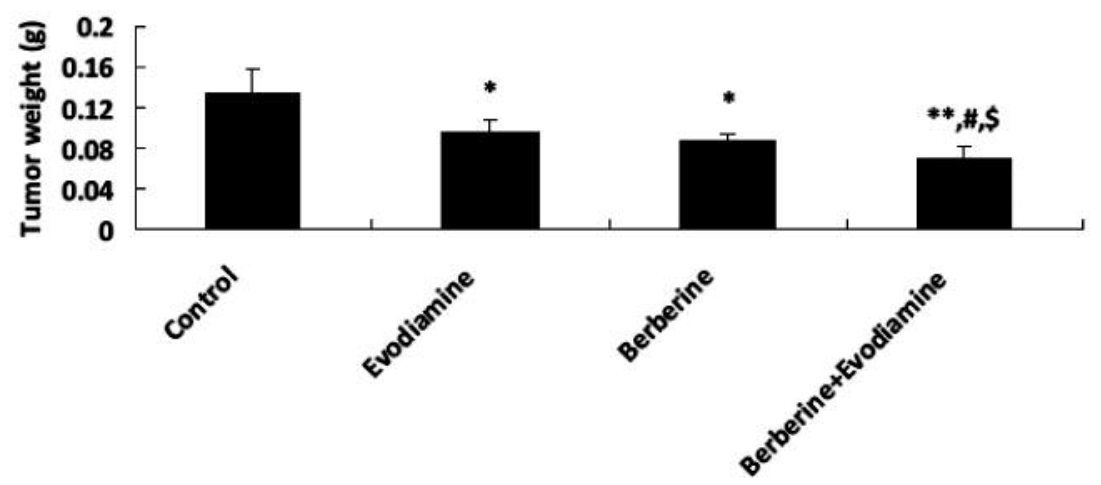

Figure 7. The body weight (A) and tumor growth curves (B) of 4 treatment and normal groups during 6 weeks $(n=5)$. (C) Tumor weights of the 4 treatment groups are shown. Values are expressed mean values $\pm S D$. ${ }^{*} p<0.05,{ }^{*} p<0.01$, evo, ber, evo + ber vs. Model; ${ }^{*} p<0.05$, \#\# $<<0.01$, evo + ber vs. evo; $\$ p<0.05$, evo + ber vs. ber.

suggesting that p53 is involved in the compound combination-induced apoptotic process.

Synergistic drug combinations have been explored to achieve one or more favorable outcomes, such as enhanced efficacy, decreased dosage at an equal or increased level of efficacy (25). Previously, berberine was evaluated for its ability to sensitize cancer cells to the anti-cancer effects of doxorubicin (26). In another study, evodiamine augmented the therapeutic efficacy of gemcitabine (27). In this study, 25 $\mu \mathrm{mol} / 1$ berberine or $15 \mu \mathrm{mol} / 1$ evodiamine alone resulted in 


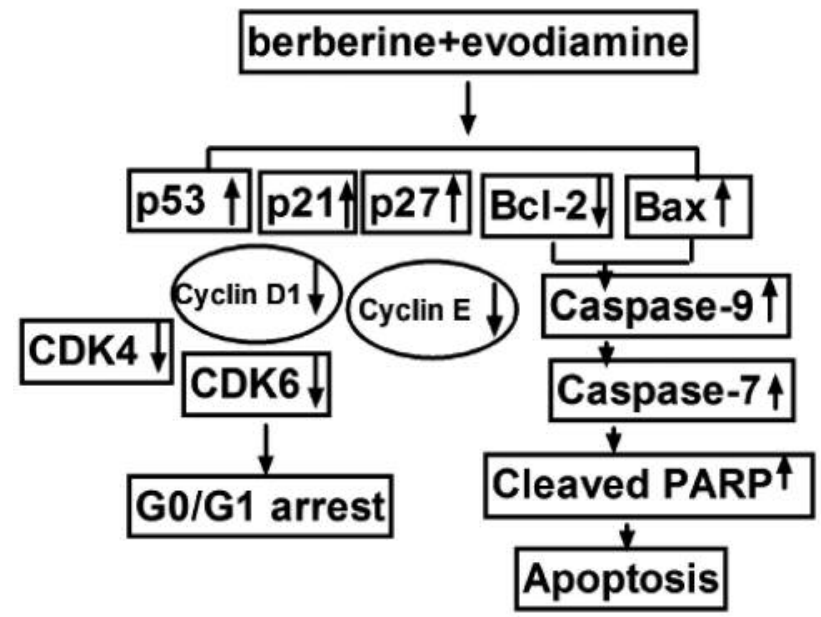

Figure 8. A schematic diagram illustrating the molecular mechanism underlying how berberine (ber) and evodiamine (evo) inhibit MCF-7 breast cancer cell proliferation. The mechanisms of ber and evo might involve the expression of cyclins, CDKs, Bcl-2 and the activation of the caspase-9 apoptotic pathway, leading to reduced cell proliferation.

moderate or no changes in the expression of proteins related to cell-cycle arrest or induction of apoptosis. However, the same doses of the combination treatment elicited synergistic effects on the up-regulation or down-regulation of these proteins, effectively inhibiting cell growth.

\section{Conclusion}

Through this study, the combination of berberine and evodiamine, which are found in the TCM formula Zuo-JinWan, was used to treat MCF-7 cells in vitro and in vivo. This combination treatment acts synergistically against the human breast cancer MCF-7 cells by inducing cel-cycle arrest and apoptosis. These promising results suggest that the TCM formula-based combination of berberine and evodiamine exhibits significant anti-breast cancer activity and demonstrated that TCM formulas might represent valuable resources for the future study of synergistic natural product treatments for cancer.

\section{Conflicts of Interest}

The Author (s) declare that they have no competing interests.

\section{Acknowledgements}

This research was supported by the Key Program of National Natural Science Funds of China (No. 81330084), the Program for Professor of Special Appointment (Eastern Scholar) at the Shanghai Institutions of Higher Learning (No.201289), and the Institutes of Shanghai Municipal Education Commission (No. E03008).

\section{References}

1 Siegel R, Naishadham D and Jemal A: Cancer statistics. CA Cancer J Clin 63(1): 11-30, 2013.

2 Gordaliza M: Natural products as leads to anticancer drugs. Clin Transl Oncol 9(2): 67-776, 2007.

3 Wagner $\mathrm{H}$ and Ulrich-Merzenich $\mathrm{G}$ : Approaching a new generation of phytopharmaceuticals, Phytomedicine. Synergy Res 16: 97-110, 2009.

4 Liu YH, Kong JM, Chia LS and Goh NK: Synergistic effect of Traditional Chinese Medicine. Asian Journal of Chemistry 19(2): 867-882, 2007.

5 Tang QF, Sun J, Yu H, Shi XJ, Lv R, Wei HC and Yin PH: The Zuo Jin Wan Formula Induces Mitochondrial Apoptosis of Cisplatin-Resistant Gastric Cancer Cells via Cofilin-1. Evid Based Complement Alternat Med 2016: 8203789, 2016.

6 Chou ST, Hsiang CY, Lo HY, Huang HF, Lai MT, Hsieh CL, Chiang SY and Ho TY: Exploration of anti-cancer effects and mechanisms of Zuo-Jin-Wan and its alkaloid components in vitro and in orthotopic HepG2 xenograft immunocompetent mice. BMC Complement Altern Med 17(1): 121, 2017.

7 Sui H, Liu X, Jin BH, Pan SF, Zhou LH, Yu NA, Wu J, Cai JF, Fan ZZ, Zhu HR and Li Q: Zuo-Jin-Wan, a Traditional Chinese Herbal Formula, Reverses P-gp-mediated MDR in vitro and in vivo. Evid Based Complement Alternat Med 2013: 957078, 2013.

8 Xu L, Qi Y, Lv L, Xu Y, Zheng L, Yin L, Liu K, Han X, Zhao $\mathrm{Y}$ and Peng J: In vitro anti-proliferative effects of Zuo-Jin-Wan on eight kinds of human cancer cell lines. Cytotechnology 66(1): 37-50, 2014.

9 Du J, Sun Y, Wang XF, Lu YY, Zhou QM and Su SB: Establishment of an experimental breast cancer ZHENG model and curative effect evaluation of Zuo-Jin-Wan. Evid Based Complement Alternat Med 2013: 324732, 2013.

10 Yin J, Zhang HJ and Ye JP: Traditional Chinese Medicine in Treatment of Metabolic Syndrome, Endocrine Metabolism Immune Disorder. Drug Targets 8(2): 99-111, 2008.

11 Vuddanda PR, Chakraborty S and Singh S: Berberine: a potential phytochemical with multispectrum therapeutic activities. Expert Opinion Investing Drugs 19(10): 1297-1307, 2010.

12 Guo Y, Pope C, Cheng X, Zhou H and Klaassen CD: Doseresponse of berberine on hepatic cytochromes P450 mRNA expression and activities in mice. J Ethnopharmacol 138(1): 111118, 2011.

13 Kobayashi Y, Nakano Y, Kizaki M, Hoshikuma K, Yokoo Y and Kamiya T: Capsaicin-like anti-obese activities of evodiamine from fruits of Evodia rutaecarpa, a vanilloid receptor agonist. Planta Med 67(7): 628-633, 2001.

14 Dai JP, Li WZ, Zhao XF, Wang GF, Yang JC, Zhang L, Chen XX, $\mathrm{Xu}$ YX and Li KS: A Drug Screening Method Based on the Autophagy Pathway and Studies of the Mechanism of Evodiamine against Influenza AVirus. PLoS One 7(8): e42706, 2012.

15 Rang WQ, Du YH, Hu CP, Ye F, Xu KP, Peng J, Deng HW and Li YJ: Protective effects of evodiamine on myocardial ischemia-reperfusion injury in rats. Planta Med 70(12): 11401143, 2004.

16 Lin H, Tsai SC, Chen JJ, Chiao YC, Wang SW, Wang GJ, Chen $\mathrm{CF}$ and Wang PS: Effects of evodiamine on the secretion of testosterone in rat testicular interstitial cells. Metabolism 48(12): 1532-1535, 1999. 
17 Jiang J and Hu C: Evodiamine: a novel anti-cancer alkaloid from Evodia rutaecarpa. Molecules 14(5): 1852-1859, 2009.

18 Tillhon M, Guamán Ortiz LM, Lombardi P and Scovassi AI: Berberine: new perspectives for old remedies. Biochem Pharmacol 84(10): 1260-1267, 2012.

19 Takada Y, Kobayashi Y and Aggarwal BB: Evodiamine abolishes constitutive and inducible NF-kappaB activation by inhibiting IkappaBalpha kinase activation, thereby suppressing NFkappaB-regulated antiapoptotic and metastatic gene expression, up-regulating apoptosis, and inhibiting invasion. J Biol Chem 280(17): 17203-17212, 2005.

20 Pandey MK, Sung B, Kunnumakkara AB, Sethi G, Chaturvedi $\mathrm{MM}$ and Aggarwal BB: Berberine modifies cysteine 179 of IkappaBalpha kinase, suppresses nuclear factor-kappaBregulated antiapoptotic gene products, and potentiates apoptosis. Cancer Res 68(13): 5370-5379, 2008.

21 Zhang T, Qu S, Shi Q, He D and Jin X: Evodiamine induces apoptosis and enhances TRAIL-induced apoptosis in human bladder cancer cells through mTOR/S6K1-mediated downregulation of Mcl-1. Int J Mol Sci 15(2): 3154-3171, 2014

22 Kuo CL, Chi CW and Liu TY: Modulation of apoptosis by berberine through inhibition of cyclooxygenase-2 and Mcl-1 expression in oral cancer cells. In Vivo 19(1): 247-252, 2005
23 Kinghom AD, Chin YW and Swanson SM: Discovery of natural product anticancer agents from biodiverse organisms. Curr Opin Drug Discov Devel 12(2): 189-196, 2009.

24 Ling CD, Yue XQ and Ling C: Three advantages of using traditional Chinese medicine to prevent and treat tumor. J Integr Med 12(4): 331-335, 2014.

25 Tang HY, Shih A, Cao HJ, Davis FB, Davis PJ and Lin HY: Resveratrol-induced cyclooxygenase- 2 facilitates $\mathrm{p} 53$-dependent apoptosis in human breast cancer cells. Mol Cancer Ther 5(8): 2034-2042, 2006.

26 Jia J, Zhu F, Ma X, Cao Z, Li Y and Chen YZ: Mechanisms of drug combinations: interaction and network perspectives. Nat Rev Drug Discov 8(2): 111-128, 2009.

27 Wei WT, Chen H, Wang ZH, Ni ZL, Liu HB, Tong HF, Guo HC, Liu DL and Lin SZ: Enhanced antitumor efficacy of gemcitabine by evodiamine on pancreatic cancer via regulating PI3K/Akt pathway. Int J Biol Sci 8(1): 1-14, 2012.

Received July 10, 2017

Revised September 27, 2017

Accepted September 28, 2017 